\title{
Excitonic effects in band-edge luminescence of semiconductors at room temperatures
}

\author{
A.V. Sachenko, Yu.V. Kryuchenko \\ Institute of Semiconductor Physics of NASU, prospect Nauki 45, Kiev, 03028, Ukraine
}

\begin{abstract}
A theoretical analysis is developed for ascertaining the influence of exciton states on edge luminescence in different semiconductors at high temperatures and high levels of excitation. Screening effects and the Mott transition for excitons have been taken into account using simple relations obtained with a variational method. Dependencies of luminescence quantum efficiency on excitation level are discussed. A mechanism of exciton non-radiative annihilation due to Auger recombination via deep impurity levels is analysed as well. It is shown that the probability of this process decreases with an energy of exciton binding.
\end{abstract}

Keywords: room-temperature exciton luminescence, high excitation levels, quantum efficiency, exciton nonradiative annihilation, Auger mechanism.

Paper received 10.09.99; revised manuscript received 15.12.99; accepted for publication 14.03.00.

\section{Introduction}

As was argued for the first time in 1967 by Nolle [1] at certain conditions the exciton contribution into edge luminescence in semiconductors can be the principal one not only at low (liquid helium or nitrogen) temperatures, but at room temperatures, too. In this case the radiative recombination is suppressed not only by non-radiative multi-phonon processes of energy dissipation, but Auger recombination of carriers, too. Moreover, along with conventional band-to-band Auger recombination of carriers a specific exciton Auger-type annihilation via deep impurity levels contributes to non-radiative dissipation of energy [2-5]. As it was shown in [2,3,6], this recombination mechanism can dominate in silicon, especially at sufficiently large doping levels or high laser excitation. It is expected also, that this mechanism should manifest itself in other semiconductors.

In the present work some properties of edge luminescence have been considered for the case of high temperatures when exciton dissociation time is much less than characteristic exciton annihilation time. If, furthermore, the binding energy of exciton ground state becomes less than thermal energy $k T$, the isolated lines of free excitons cannot be observed in optical spectra in principle. Nevertheless, even under these conditions the excitons can contribute substantially to the luminescence and determine its intensity at least for concentrations of excess carriers lower than the critical one corresponding to the exciton Mott transition. We have considered this effect in a simple model, which allows to take into account and evaluate the influence of above mentioned Auger-type mechanism of exciton non-radiative annihilation via deep impurity levels on quantum efficiency of luminescence both in direct- and indirect-gap semiconductors.

\section{Formulation of the problem}

Since quantum efficiency of radiative recombination should be determined in a wide range of excitation intensities and doping levels, it is necessary to take into account the effect of Coulomb potential screening between electron and hole at sufficiently large concentrations of free carriers. It can be done by the analogy with [7] on the base of variational method. A ground state wave function of the Wannier-Mott exciton can be approximated in the framework of this method by the following expression:

$\psi(r)=A \exp \left(-\frac{r}{a}\right)$,

where $r$ is the radial coordinate, $A$ is the normalization constant and $a$ is the variational parameter. Potential energy of the electron-hole interaction is taken in a standard form of screened Coulomb potential: 


\section{A.V. Sachenko, Yu.V. Kryuchenko: Excitonic effects in band-edge luminescence..}

$$
V(r)=-\frac{q^{2}}{\varepsilon \cdot r} \exp \left(-\frac{r}{r_{D}}\right)
$$

where $q$ is the electron (hole) charge, $\varepsilon$ is the static dielectric constant, $r_{D}=\left(\varepsilon k T / 4 \pi q^{2}(n+p)\right)^{1 / 2}$ is the screening radius in a nondegenerate case. For further consideration it is also convenient to introduce the following standard designations: the binding energy of the Wannier-Mott exciton ground state in the absence of screening, $E_{0}=-\frac{\mu q^{4}}{2 \varepsilon^{2} \hbar^{2}} ;$ the corresponding exciton Bohr radius, $a_{B}=\frac{\hbar^{2} \varepsilon}{\mu q^{2}}$; the reduced effective exciton mass, $\mu=m_{e} \times \times m_{h} /\left(m_{e}+m_{h}\right)$. Then from the minimum condition for the exciton ground state energy with account for screening one can obtain the following equation for variational parameter $\delta=a / a_{B}$ :

$$
\delta^{3}-\left(2 \beta^{2}-3 \beta\right) \delta^{2}-\left(\beta^{3}-3 \beta\right) \delta+\beta^{3}=0,
$$

where $\beta=2 r_{D} / a_{B}$.

Finally, the expression for the exciton binding energy in the case of screening reduces to the following one:

$$
E_{x}=E_{0}\left(\frac{2}{\delta(1+\delta / \beta)^{2}}-\frac{1}{\delta^{2}}\right)
$$

The condition $E_{x}=0$ determines the point of exciton Mott transition. It follows from Eqs (3) and (4) that this transition occurs at $\beta \cong 2.133$. It should be noticed that obtained solution is valid for both direct- and indirect-gap semiconductors. From the screening radius value at the transition point $\dot{r}_{D} \cong 1,067 a_{B}$ one can easily obtain the critical concentration of carriers, at which exciton Mott transition takes place. For example, in the framework of our consideration the critical concentration for the case of silicon at $T=300 \mathrm{~K}$ turns out to be $\approx 8.3 \cdot 10^{17} \mathrm{~cm}^{-3}$, which is somewhat lower than usually used value $1.03 \cdot 10^{18} \mathrm{~cm}^{-3}$.

It should be noticed also that the condition $\beta \cong 2.133$ for the exciton Mott transition is quite general one and thus is valid at much lower temperatures, too. The only difference, in comparison to the case of high temperatures, is that it is necessary to take into account possible carriers degeneracy, which changes the expression for $r_{D}$ in appropriate way. For instance, in the case of the degeneracy in electron subsystem one has to use the follow-

ing expression: $r_{D}^{-2}=\frac{2 \sqrt{2}}{\sqrt{\pi}} \frac{q^{2} m_{n}^{3 / 2}(k T)^{1 / 2}}{\varepsilon \hbar^{3}} F_{1 / 2}^{\prime}\left(\frac{E_{F n}}{k T}\right)$, where $m_{n}$ is the effective density-of-states mass for electrons, $F_{1 / 2}^{\prime}(z)$ the first derivative of the Fermi-Dirac inte- gral of the one half order over argument, $E_{F n}$ the Fermi energy for electrons measured from the conduction-band edge.

Expression (4) can be approximated with a good degree of accuracy by straightforward relation:

$E_{x} \cong E_{0}\left(1-\frac{2}{\beta}\right)^{2}=E_{0}\left(1-\frac{a_{B}}{r_{D}}\right)^{2}$,

which is typically used in literature.

Fig. 1 illustrates the dependencies of variational parameter $\delta$ and exciton binding energy $E_{x}$ on $\beta$. For a comparison, binding energy dependencies are build both with exact formula (4) and approximate one (5). As one can see from this figure the approximate expression (5) actually gives results very close to (4) in the entire $\beta$ range up to the Mott transition.

In line with approach of $[8,9]$, neglecting the effects of higher orders [10], the kinetic equations for continuous flow of excess electron-hole pairs generated by monochromatic laser excitation as well as for the flux of

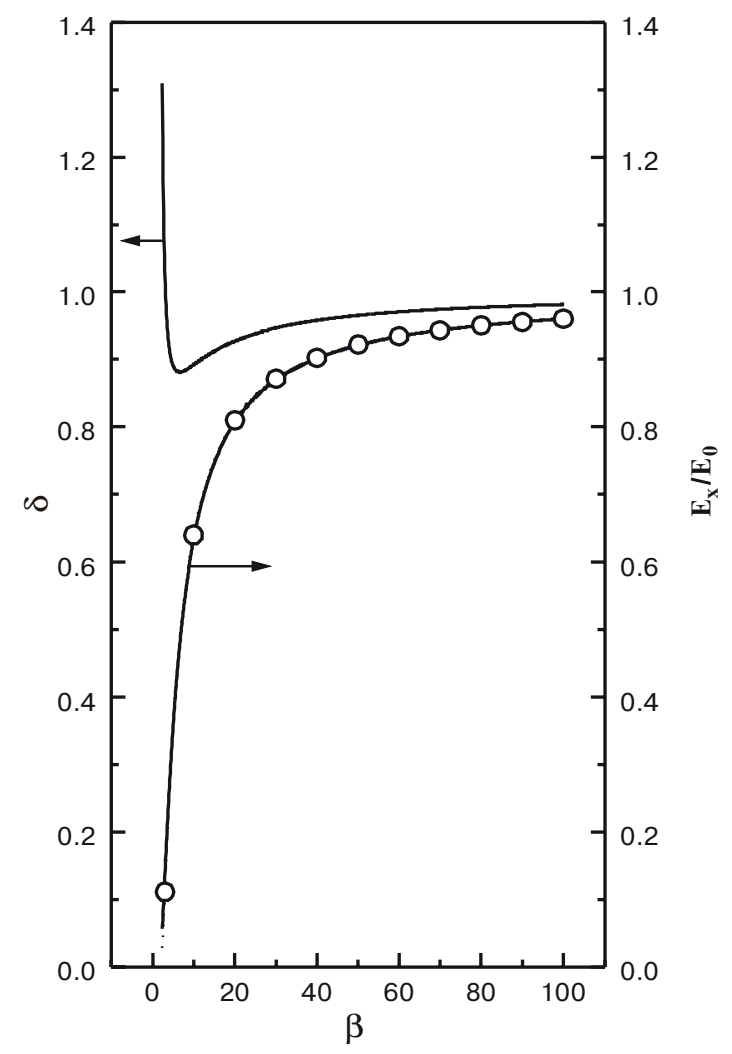

Fig. 1. Dependencies of a variational parameter $\delta$ and an exciton binding energy $E_{x}$ on the screening parameter $\beta$. Solid $E_{x}(\beta)$ curve is built using the exact formula (4), while the dashed with circles one the approximate formula (5). $E_{0}$ is the exciton binding energy when screening is absent. 


\section{A.V. Sachenko, Yu.V. Kryuchenko: Excitonic effects in band-edge luminescence...}

excitons formed due to the Coulomb interaction between carriers can be represented in the following form:

$$
\begin{aligned}
& \frac{\partial \Delta p}{\partial t}=D_{A} \frac{\partial^{2} \Delta p}{\partial x^{2}}- \\
& -\left[\frac{1}{\tau_{r n}}+A_{i}\left(n_{0}+\Delta n\right)+C_{n}\left(n_{0}+\Delta n\right)^{2}+\right. \\
& \left.+C_{p}\left(n_{0}+\Delta n\right) \Delta p\right] \Delta p-\gamma_{1}\left(\left(n_{0}+\Delta n\right) \Delta p-n^{*} n_{x}\right)+ \\
& +\alpha I_{0} \exp (-\alpha x), \\
& \frac{\partial \Delta n_{x}}{\partial t}=D_{x} \frac{\partial^{2} \Delta n_{x}}{\partial x^{2}}-\frac{\Delta n_{x}}{\tau_{x}}+\gamma_{1}\left(\left(n_{0}+\Delta n\right) \Delta p-n^{*} n_{x}\right),
\end{aligned}
$$

where $\Delta n$ and $\Delta p$ are the bulk concentrations of excess electrons and holes inside a semiconductor, $\tau_{r n}$ is the Shockley-Reed-Hall life time, $A_{i}$ is the constant of radiative interband recombination, $C_{n}$ and $C_{p}$ are the parameters of band-to-band Auger recombination for electrons and holes respectively, $\gamma_{l}$ is the probability of electron-hole pairs association into the excitons, $n^{*}=n^{* *} \exp \left(-E_{x} / k T\right)$ is the characteristic concentration of excitons in the case of thermodynamic equilibrium, $n^{* *}=N_{c} N_{v} / N_{x}, N_{i}=g_{i}\left(2 \pi m_{i} k T / h^{2}\right)^{3 / 2}$ is the effective density of states $(i=c, v, x), g_{i}$ is the degeneracy factor, $m_{i}$ is the effective density-of-state mass in the cases of electrons and holes, $m_{x}=m_{c}+m_{v}$ in the case of exciton, $\tau_{x}$ is the exciton radiative annihilation time, $n_{x}$ and $\Delta n_{x}$ are the total and excess exciton concentrations in crystal, $I_{0}$ is the intensity of exciting monochromatic light transmitted through the surface into a crystal in the spectral region of fundamental absorption, $\alpha$ is the absorption coefficient, $D_{A}$ and $D_{x}$ are the ambipolar diffusion coefficients for carriers and diffusion coefficient for excitons, respectively.

For definiteness, an $n$-type semiconductor with an equilibrium bulk electron concentration $n_{0}$ is considered. It is supposed also that the hole concentration at an external excitation exceeds substantially equilibrium hole concentration in the absence of excitation and thus $\Delta n_{x} \cong n_{x}$.

The characteristic reduced time of exciton annihilation, $\tau_{x}$, can be found from the rule for the sum of reciprocal times $\tau_{x}^{r}$ and $\tau_{x}^{n}$ of radiative and nonradiative exciton annihilation:

$\frac{1}{\tau_{x}}=\frac{1}{\tau_{x}{ }^{r}}+\frac{1}{\tau_{x}{ }^{n}}$.

The system of equations (6)-(8) should be solved together with the following boundary conditions for the flows:

$$
j_{p}(x=0, d)=-\left.D_{A} \frac{d \Delta p}{d x}\right|_{x=0, d}=\mp S_{0(d)} \Delta p(x=0, d),(9)
$$

$$
\begin{aligned}
& j_{x}(x=0, d)=-\left.D_{x} \frac{d \Delta n_{x}}{d x}\right|_{x=0, d}= \\
& =\mp S^{x} 0(d) \Delta n_{x}(x=0, d),
\end{aligned}
$$

where $S_{O(d)}$ and $S^{x}{ }_{0(d)}$ are the effective rates of electronhole recombination and exciton annihilation at the surfaces $x=0, d$ of a crystal.

As a rule, at high temperatures, the time of exciton dissociation $\left(\gamma_{1} n^{*}\right)^{-1}$ is substantially smaller than exciton annihilation time, $\tau_{x}$. Indeed, in accordance with estimates of $[1,8,11]$ the dissociation time of free excitons is about $10^{-12} \mathrm{~s}$, which is several orders of magnitude smaller than typical $\tau_{x}$ values in all semiconductor materials. At such conditions it is possible to refer the excitons only in statistical sense, i.e their concentration $n_{x}$ can become substantial only at high excitation levels due to a quadratic growth of $n_{x}$ with concentration of nonequilibrium carriers. The relaxation of nonequilibrium excitons has to occur in this case within characteristic time which may differ substantially from exciton annihilation time $\tau_{x}$. For example, at linear response of a system with regard to major carriers, i.e. when $\Delta n<<n_{0}$, the effective relaxation time of excitons turns out to be the same as of excess holes. This is expressed by the following formula:

$\tau^{*}=\frac{\left(n_{0}+n^{*}\right) \tau_{x} \tau_{r}^{*}}{n_{0} \tau_{r}^{*}+n^{*} \tau_{x}}$,

where $\tau_{r}^{*}$ is the effective hole lifetime when all electronhole recombination channels are taken into account. If $n_{0}<<n^{*}$ and $\tau_{r}^{*} n_{0}<<\tau_{x} n^{*}$ then $\tau^{*} \approx \tau_{r}^{*}$. In the case of $n_{0}<<n^{*}$ and $\tau_{r}^{*} n_{0}>>\tau_{x} n^{*}$ the effective relaxation time should be $\tau^{*} \approx \tau_{x} n^{*} / n_{0}$.

In the considered model the approximate solution of Eq. (7) has the following form:

$n_{x} \cong \frac{1}{n^{*}}\left(n_{0}+\Delta n\right) \Delta p$

Then, combining Eqs (6) and (7) as well as taking into account Eq. (12), one can obtain for the case of stationary process at high excitation levels, i.e. when $\Delta n \approx \Delta p>n_{0}$, the following equation:

$$
\begin{aligned}
& D_{A} \frac{d^{2} \Delta p}{d x^{2}}-\left[\frac{1}{\tau_{r n}}+A_{i}\left(n_{0}+\Delta p\right)+\right. \\
& \left.+C_{n}\left(n_{0}+\Delta p\right)^{2}+C_{p}\left(n_{0}+\Delta p\right) \Delta p\right] \Delta p+ \\
& +\frac{D_{x}}{n^{*}}\left[\left(n_{0}+2 \Delta p\right) \frac{d^{2} \Delta p}{d x^{2}}+2\left(\frac{d \Delta p}{d x}\right)^{2}\right]- \\
& -\frac{1}{n^{*} \tau_{x}}\left(n_{0}+\Delta p\right) \Delta p+\alpha I_{0} \exp (-\alpha x)=0 .
\end{aligned}
$$




\section{A.V. Sachenko, Yu.V. Kryuchenko: Excitonic effects in band-edge luminescence..}

Solution of Eq. (13) along with boundary conditions (9) allows to obtain $\Delta p$ as a function of the intensity of exciting light. It should be emphasized that in the considered case the radiative and nonradiative exciton annihilation, which is described by the fourth term in Eq. (13), actually is equivalent to additional quadratic recombination of electron-hole pairs.

Radiation lifetime of excitons can be evaluated from the principle of detailed equilibrium between absorption and radiation in the region of exciton absorption. For example, the estimates in $[1,6]$ demonstrate that for indirect-gap semiconductors it amounts to $10^{-3} \div 10^{-4} \mathrm{~s}$, while for direct-gap semiconductors to $\sim 10^{-9} \div 10^{-10}$ with the tendency to decrease as the band-gap grows.

To estimate exciton nonradiative annihilation time we have used a simple model described earlier in $[2,6]$. This model is based on the fact that the Wannier-Mott exciton forms a region with a large local carrier density $n_{L} \sim\left(4 \pi a_{B}^{3} / 3\right)^{-1}$. This concentration is sufficiently high to initiate a pronounced Auger recombination process, especially when deep impurities are involved. Really, in conventional materials just this Auger recombination is responsible for exciton nonradiative annihilation. As a result the time of exciton nonradiative annihilation can be expressed by the following formula:

$$
\tau_{x}^{n}=\left(n_{L} G N_{t}\right)^{-1}
$$

where $G$ is the constant of Auger recombination process with impurity participation, $N_{t}$ is the concentration of deep impurities. If the condition

$$
\tau_{x}^{r} n_{L} G N_{t}<<1
$$

is realized then the exciton nonradiative recombination can be neglected in comparison with exciton radiative annihilation. It is clear from (15) that the role of exciton nonradiative recombination via Auger mechanism becomes less important with decrease of the concentration of deep impurities. The same effect is expected with $n_{L}$ decrease, i.e. when exciton binding energy becomes smaller.

On the other hand, taking into account excitonic transitions, the absorption coefficient in direct-gap semiconductors at the absorption edge can be written in accordance with [12] in the following form:

$K_{x}\left(E_{g}\right)=2 \pi B \sqrt{E_{0}}$,

where $B$ is the same normalizing factor as in frequency dependence of absorption coefficient when excitonic effects are neglected:

$K(h v)=B\left(h v-E_{g}\right)^{1 / 2}$,

i.e. $B=2 q^{2} \mu^{3 / 2} /\left(\varepsilon^{1 / 2} c h^{2} m_{n}\right), c$ is the light velocity and $m_{n}$ is the effective electron mass.

The probability of radiative annihilation of excitons is proportional to $K_{x}\left(E_{g}\right)$. From the relation $K_{x}\left(E_{g}\right) \tau_{x}^{n} \sim \varepsilon^{1 / 2} / \mu m_{n}$ it follows that the ratio of exciton radiative annihilation to exciton nonradiative recombination turns out to be higher for more «shallow» exciton, i.e. for lower $\mu$ and $m_{n}$ values, which is one confirmation more for the validity of our previous conclusions. At the same time the exciton contribution into absorption in accordance with (16) is larger for excitons with higher binding energy.

In the Table 1 one can find the values of reduced exciton mass, exciton Bohr radius, exciton binding energy as well as $n_{M}, n_{L}, n^{*}$ and $n_{x}$ at $T=300 \mathrm{~K}$ for different semiconductors. The values of $n_{x}$ have been obtained for the case of $\Delta p=\Delta n=10^{17} \mathrm{~cm}^{-3}$.

The data in this Table are based on the information presented in $[1,6,13,14]$.

As one can see from this Table an explicit correspondence exists between some important parameters in different semiconductors in accordance with the above discussed qualitative dependencies. In particular, in such

Table 1.

\begin{tabular}{lllllllll}
\hline \hline & $E_{g}, \mathrm{eV}$ & $\mu / m_{0}$ & $a_{B}, \mathrm{~nm}$ & $E_{0}, \mathrm{meV}$ & $n_{M}, 10^{17} \mathrm{~cm}^{-3}$ & $n_{L}, 10^{18} \mathrm{~cm}^{-3}$ & $n^{*}, 10^{17} \mathrm{~cm}^{-3}$ & $n_{x}=\Delta p^{2} / n^{*}, 10^{16} \mathrm{~cm}^{-3}$ \\
\hline Si & 1.12 & 0.15 & 4.2 & 14.7 & 8.3 & 3.3 & 8 & 1.25 \\
\hline InP & 1.35 & 0.06 & 10.7 & 6.0 & 1.32 & 0.195 & 1.46 & 6.83 \\
\hline GaAs & 1.43 & 0.061 & 10.4 & 5.8 & 1.39 & 0.21 & 1.51 & 6.61 \\
\hline GaP & 2.21 & 0.128 & 4.14 & 17 & 7.3 & 3.36 & 2.98 & 3.35 \\
\hline $\mathbf{C d T e}$ & 1.47 & 0.084 & 6.9 & 9.6 & 2.88 & 0.733 & 2.11 & 4.74 \\
\hline $\mathbf{C d S e}$ & 1.74 & 0.101 & 5.57 & 12 & 4.27 & 1.38 & 2.53 & 3.95 \\
\hline $\mathbf{C d S}$ & 2.53 & 0.143 & 3.3 & 25 & 10.2 & 6.64 & 2.59 & 3.87 \\
\hline ZnTe & 2.28 & 0.13 & 3.96 & 19 & 7.73 & 3.85 & 2.83 & 3.54 \\
\hline ZnSe & 2.58 & 0.137 & 3.14 & 28 & 10.3 & 7.71 & 2.16 & 4.63 \\
\hline ZnO & 3.2 & 0.146 & 2.87 & 32 & 12 & 10.1 & 2.04 & 4.91 \\
\hline $\boldsymbol{\alpha - Z n S}$ & 3.8 & 0.179 & 2.46 & 35 & 17 & 16 & 2.46 & 4.06 \\
\hline \hline
\end{tabular}




\section{A.V. Sachenko, Yu.V. Kryuchenko: Excitonic effects in band-edge luminescence...}

semiconductors as GaAs and CdTe, where the internal quantum efficiency of radiative recombination is close to $100 \%$, excitons are the «shallow» ones and the concentration $n_{L}$ is relatively small. Values of $n_{M}$ correspond to critical concentrations of charge carriers at which these exciton Mott transitions take place.

Let us consider the case of high excitation levels providing substantial nonlinear effects in a system of nonequilibrium carriers. Then for uniform absorption of exciting light inside the crystal and negligibly small surface recombination rates at surfaces $x=0, d$ the $\Delta p$ dependence on the intensity $I_{0}$ is determined by the following formula:

$\left[\frac{1}{\tau_{r n}}+A_{i}\left(n_{0}+\Delta p\right)+C_{n}\left(n_{0}+\Delta p\right)^{2}+C_{p}\left(n_{0}+\Delta p\right) \Delta p\right] \times$

$\times \Delta p+\frac{\left(n_{0}+\Delta p\right) \Delta p}{\tau_{x} n^{* *}} \exp \left[\frac{E_{0}}{k T}\left(1-\left(\frac{2 \Delta p}{n_{M}}\right)^{.1 / 2}\right)^{2}\right]=\alpha I_{0}$

The total intensity $J_{\text {Total }}$ of band-edge luminescence can be found by calculating the following integral:

$J_{\text {Total }}=\int_{0}^{d}\left[A_{i}+\frac{1}{n^{*} \tau_{x}^{r}}\right]\left(n_{0}+\Delta p(x)\right) \Delta p(x) d x$,

while quantum efficiency $\eta$ for this process is determined by the formula

$\eta=\frac{\int_{0}^{d}\left[A_{i}+\frac{1}{n^{*} \tau_{x}^{r}}\right]\left(n_{0}+\Delta p(x)\right) \Delta p(x) d x}{I_{0}(1-\exp (-\alpha d))}$.

It should be pointed out that effect of reabsorption is not taken into account in (19) and (20). Therefore, these equations actually are valid only for a plate with thickness $d$ less than the effective reabsorption length of emitted light in a crystal.

Obtained with Eq. (18) theoretical dependencies of excess electron (hole) concentration on intensity of external laser excitation for the cases of Si, GaAs and InP are shown in Fig. 2. The parameters used to obtain these dependencies are presented in Table 2, which is based on the data of $[1,6,15]$. As one can see from the figure, high

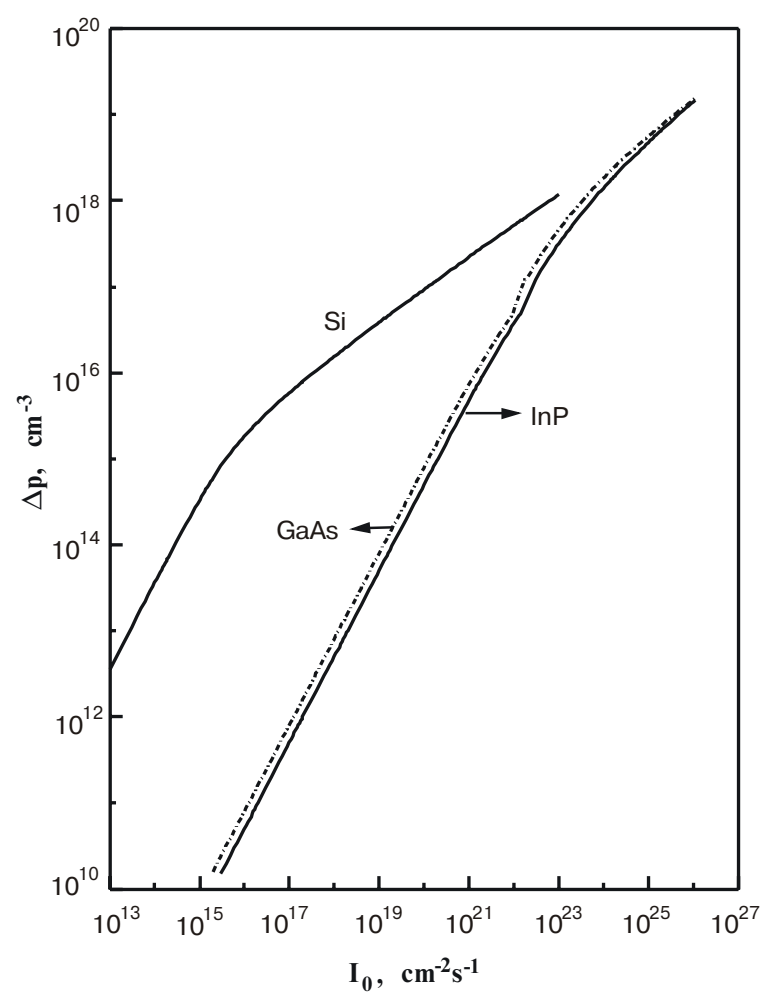

Fig. 2. Concentration of excess electron-hole pairs $\Delta p$ versus intensity of exciting light $I_{0}$ in different semiconductors.

$\Delta p$ concentrations in Si can be achieved at lower levels of excitation than in GaAs and InP. It is connected with substantially larger value of the Shokley-Reed-Hall lifetime in Si comparing to GaAs and InP.

Figs 3 and 4 demonstrate the dependencies of total photoluminescence intensity and quantum efficiency of radiative recombination on the concentration of excess electron-hole pairs in $\mathrm{Si}, \mathrm{GaAs}$ and InP. As can be seen from Fig. 3, total photoluminescence intensity in $\mathrm{Si}$ is more than three orders of magnitude weaker than in GaAs and InP for the used parameters providing the equivalent values of absorbed radiation in all three materials. In the cases of $\mathrm{GaAs}$ and InP an exciton luminescence yields a substantial contribution into the total $J$ value in the region of concentrations $\Delta p<n_{M} / 2$. In the vicinity of the exciton Mott transition, a nonmonotonic behavior in $J(\Delta p)$ dependencies is predicted. It should be particu-

Table 2.

\begin{tabular}{|c|c|c|c|c|c|c|c|c|c|c|}
\hline & $\tau_{r n}, \mathrm{~s}$ & $\tau_{x}, \mathrm{~s}$ & $A_{i}, \mathrm{~cm}^{3} / \mathrm{s}$ & $C_{p}, \mathrm{~cm}^{6} / \mathrm{s}$ & $C_{n}, \mathrm{~cm}^{6} / \mathrm{s}$ & $E_{0} / k T$ & $n^{* *}, \mathrm{~cm}^{-3}$ & $n_{M}, \mathrm{~cm}^{-3}$ & $d, \mathrm{~mm}$ & $\alpha, \mathrm{cm}^{-1}$ \\
\hline Si & $3.8 \cdot 10^{-2}$ & $10^{-3}$ & $1.5 \cdot 10^{-15}$ & $10^{-31}$ & $\begin{array}{l}2.8 \cdot 10^{-31}+ \\
+2.5 \times \\
10^{-22} \cdot \Delta p^{-1 / 2}\end{array}$ & 0.567 & $1.4 \cdot 10^{18}$ & $8.3 \cdot 10^{17}$ & 100 & 10 \\
\hline GaAs & $8 \cdot 10^{-9}$ & $4 \cdot 10^{-9}$ & $2 \cdot 10^{-10}$ & $4.6 \cdot 10^{-31}$ & $1.6 \cdot 10^{-29}$ & 0.224 & $1.89 \cdot 10^{17}$ & $1.39 \cdot 10^{17}$ & 1 & $10^{3}$ \\
\hline InP & $5 \cdot 10^{-9}$ & $4 \cdot 10^{-9}$ & $3.6 \cdot 10^{-10}$ & $8.7 \cdot 10^{-30}$ & $3.7 \cdot 10^{-31}$ & 0.232 & $1.84 \cdot 10^{17}$ & $1.32 \cdot 10^{17}$ & 1 & $10^{3}$ \\
\hline
\end{tabular}


A.V. Sachenko, Yu.V. Kryuchenko: Excitonic effects in band-edge luminescence..

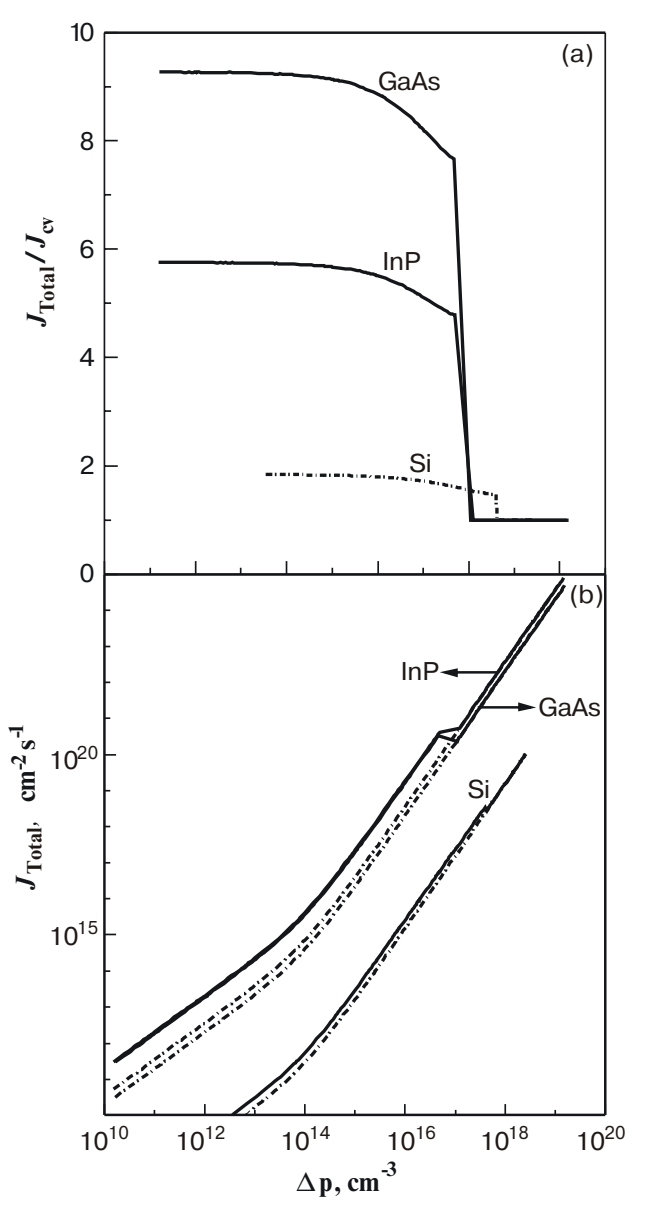

Fig. 3. (a) Ratio of total luminescence intensity $J_{\text {Total }}$ including exciton contribution to pure band-to-band luminescence $J_{c v}$ versus concentration $\Delta p$ of excess electron-hole pairs in $\mathrm{Si}$, GaAs and InP

(b) Dependencies of integrated intensity $J_{\text {Total }}$ of photoluminescence on $\Delta p$ for the same semiconductors. Dashed curves correspond to the case when exciton contribution into luminescence is not taken into account.

larly striking in the cases of GaAs, InP and other directgap semiconductors.

Comparing Figs 3 and 4 one can easily understand why silicon is worse light emitter than direct-gap semiconductors: it is due to earlier incorporation of band-toband Auger recombination with increase of excitation level. Moreover, the larger is the Shokley-Reed-Hall recombination time the earlier this effect takes place. With the used parameters the maximum quantum efficiency for the luminescence in $\mathrm{Si}$ is achieved at $\Delta p \approx 10^{15} \mathrm{~cm}^{-3}$. The corresponding intensity of total luminescence is still small in this $\Delta p$-region.

Conversely, maximum in quantum efficiency dependencies on $\Delta p$ for GaAs and InP lies in the $\Delta p$-range $10^{18} \div 10^{19} \mathrm{~cm}^{-3}$ where total luminescence intensities also achieve sufficiently high values. Like in $\mathrm{Si}$, this maximum is formed due to a substantial nonlinear increase of

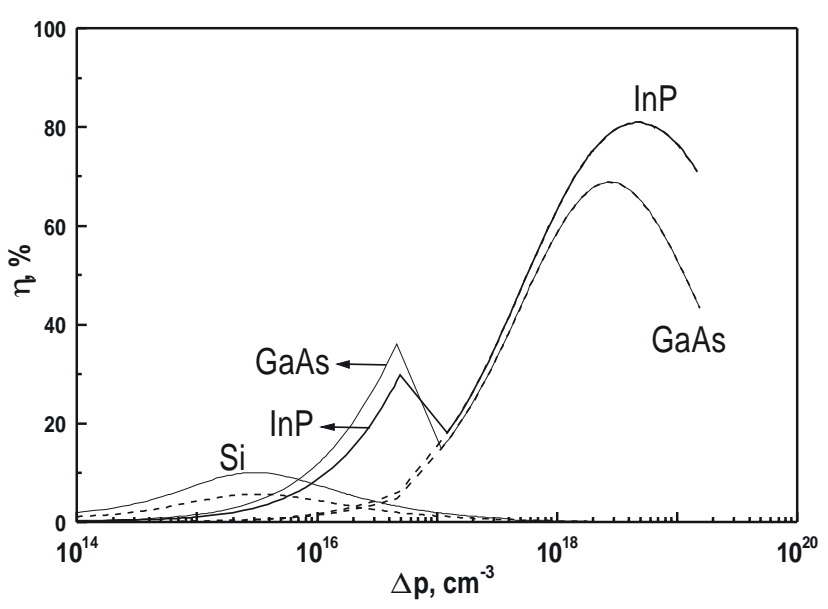

Fig. 4. Quantum efficiency of photoluminescence $\eta$ versus concentration of excess electron-hole pairs $\Delta p$ in $\mathrm{Si}$, GaAs and InP. Dashed curves illustrate the case when exciton effects are neglected.

interband Auger recombination. Quantum efficiency in these cases can be as high as $70-80 \%$ in maximum.

To increase the intensity and quantum efficiency of luminescence it would be desirable to obtain a material where the advantages of silicon and direct-gap semiconductors will be combined. Taking into account very high levels of purification and, as a result, extremely large values of the Shokley-Reed-Hall times achieved at the present time in silicon it seems real to use this material as a base. The only task is to obtain in one way or another a direct-gap material on this base which enables to increase the probability of radiative transitions both of band-toband and exciton types. For example, it can be done by application of uniaxial pressure, or by using nanocrystaline modifications as well as $\mathrm{SiGe}$ systems.

One more important point is that maximal quantum efficiency for band-edge luminescence with account for nonradiative channel of exciton annihilation is determined by the following ratio:

$\eta_{\max } \cong \frac{A_{i}+1 / n^{*} \tau_{x}^{r}}{A_{i}+1 / n^{*} \tau_{x}^{r}+n_{L} G N_{t} / n^{*}}$.

Therefore, e.g. for GaAs $\eta_{\max } \approx 1$ in the case of $G N_{t}<<10^{-9} \mathrm{~cm}^{3} \cdot \mathrm{s}^{-1}$. I.e. to achieve $\eta \approx 1$ at $N_{t} \approx 10^{15} \mathrm{~cm}^{-3}$ the Auger constant has to be $G<<10^{-24} \mathrm{~cm}^{6} \cdot \mathrm{s}^{-1}$, which is quite possible to obtain in this material.

\section{Conclusions}

In the present work an attempt has been made to evaluate within simple approximations the role of excitons in bandedge luminescence of semiconductors at high temperatures and high levels of excitation. In fact, only qualitative tendencies are analyzed and thus further theoretical 


\section{A.V. Sachenko, Yu.V. Kryuchenko: Excitonic effects in band-edge luminescence...}

and experimental investigations are necessary for more detailed quantitative analysis. Nevertheless, it is shown quite definitely that the contribution of exciton radiative transitions into the total luminescence is, as a rule, comparable or even exceeds the contribution of conventional band-to-band radiative transitions even at room temperatures.

Peculiarities in dependencies of quantum efficiency and luminescence intensity on excitation level are considered and analysed both for direct and indirect-gap semiconductors. A contribution of nonradiative channel of exciton annihilation into the total recombination rate is evaluated, too. Obviously, in a number of cases this channel is responsible for the formation in a subsurface region of crystals of the so-called «dead» layer for exciton radiative transitions and exciton luminescence from this region.

\section{References}

1. E.L. Nolle. O recombinazii cherez eksitonnye sostoyaniya $v$ poluprovodnikakh (On the recombination via exciton states in semiconductors)// Fizika Tverdogo Tela, 9 (1), pp. 122-128 (1967) (in Russian)

2. A. Hangleiter. Nonradiative recombination via deep impurity levels in silicon: Experiment // Phys. Rev. B, 35 (17), pp. 9149-9160 (1987)

3. A. Hangleiter. Nonradiative recombination via deep impurity levels in semiconductors: The exciton Auger mechanism // Phys. Rev. B, 37 (5), pp. 2594 -2604 (1988)

4. B.L. Gel'mont, V.A. Kharchenko and I.N. Yassievich. Ozherekombinaziya eksitonno-primesnykh kompleksov (An $\mathrm{Au}$ ger recombination of exciton -impurity complexes) // Fizika Tverdogo Tela, 29 (8), pp. 2351-2360 (1987) (in Russian)

5. B.L. Gel'mont, N.N. Zinoviev, D.I. Kovalev, V.A. Kharchenko, I.D. Yaroshetskii and I.N. Yassievich.
Ozhe-rekombinaziya svyazannykh eksitonov, induziruemaya akusticheskimi fononami (An Auger recombination of bound excitons induced by ultrasonic phonons) // Zhurnal Teoreticheskoi i Eksperimental'noi Fiziki, 94 (3), pp. 322-335 (1988) (in Russian)

6. A.V. Sachenko, A.P. Gorban' and V.P. Kostylyov. Excitonenhanced recombination in silicon at high concentrations of charge carriers // Semiconductor Physics, Quantum Electronics \& Optoelectronics, 3 (1), pp. 5-10 (2000)

7. Yu. Vaitkus, L. Valkunas, Yu. Vischakas and E. Skaistis. O vliyanii svobodnykh nositelei na eksitonnuyu luminescenciyu $\mathrm{v}$ poluprovodnikakh (On the influence of free carriers on exciton luminescence in semiconductors) // Litovskii fizicheskii sbornik, 16 (6), pp. 813-823 (19760 (in Russian)

8. A.V. Sachenko, V.A. Tyagai and A.G. Kundzich. Exciton luminescence in semiconductors. Surface recombination and space-charge-layer effects // Phys. Stat. Sol., B88, pp. 797804 (1978)

9. R. Corkish, S.-P. Chan Daniel and M.A Green. Excitons in silicon diodes and solar cells: A three-particle theory // J. Appl. Phys., 79 (1), pp. 195-203 (1993)

10. C.B. a la Guillaume, J.-M. Debever and F. Salvan. Radiative recombination in highly excited CdS // Phys. Rev., 177 (2), pp. 567-580 (1969)

11. M.A. Green. Excitons in silicon solar cells: room temperature distributions and flows. In: Proceedings of $2^{\text {nd }}$ World Conference and Exhibition on Photovoltaic Solar Energy Conversion, 6-10 July 1998, Vienna, Austria, pp. 74-76 (1998)

12. R.J. Elliot. Intensity of optical absorption by excitons // Phys. Rev.,108 (6), pp. 1384-1389 (1957)

13. V.I. Gavrilenko, A.M. Grekhov, D.V. Korbutyak and V.G. Litovchenko. Opticheskie svoistva poluprovodnikov. Spravochnik, (Optical properties of semiconductors. The handbook) Kiev, Naukova Dumka, 608 p. (1987) (in Russian)

14. D.E. Kane and R.M. Swanson. The effect of excitons on apparent band gap narrowing and transport in semiconductors// J. Appl. Phys., 73 (3), pp. 1193-1197 (1993)

15. G. Augustine, A. Rohatgi, N.M. Jokerst. Base doping optimization for radiation-hard $\mathrm{Si}, \mathrm{GaAs}$, and $\mathrm{InP}$ solar cells // IEEE Trans. Electron Dev., ED39 (10), pp.2395-2400 (1992) 